\title{
Outline Editing Software: CAD-Reader
}

\author{
Jiming Tan ${ }^{1, ~ a}$, Lijun Cui ${ }^{2, ~ b}$, Shoujun Qi ${ }^{3, ~ c}, X i n \mathrm{He}^{4, \mathrm{~d}}$ \\ ${ }^{1}$ No. 1310 Dagu South Road, Hexi District, Tianjin University of education and technology, Tianjin \\ ${ }^{2}$ No. 1310 Dagu South Road, Hexi District, Tianjin University of education and technology, Tianjinx \\ ${ }^{3}$ MAKINO(China)Co. Ltd \\ ${ }^{4}$ No. 1310 Dagu South Road, Hexi District, Tianjin University of education and technology, Tianjin \\ a13001318986@163.com , b13920934899@163.com, c18920063486@163.com, 514459221@qq.com
}

Keywords: SIEMENS profile editing software CAD-Reader, numerical control machining

\begin{abstract}
The paper studies a tool for turning drawings directly into processing programs -SIEMENS profile editing software CAD-Reader. CAD-Reader can convert the specified outline in the DXF drawing into NC machining procedures in a specified programming format, the operator eliminates the pain of coding. It makes CNC machining easier to learn.
\end{abstract}

\section{INTRODUCTION}

NC machining of high technical content and relates to many aspects, especially in terms of efficiency, NC programming for high speed cutting application, NC process program normalization and standardization is more prominent. The high efficiency of NC machining is related to the technology management model of the enterprise itself to a great extent. Standard Specification for NC machining program reflects the enterprise's own CNC machining technology application level to a certain extent, diversification to constrain the NC program by the standard, improve the quality of the tool path, such as indicating the location datum, tool datum, coordinate system, cutting tools and cutting parameters in the process of standardization documents; programming for multiple programming processing, from the 2D contour of 3D surface processing, fixed cycle, tool compensation and tool path processing strategies; based on typical parts processing experience, build the NC program template standardized and can greatly improve the processing efficiency and product quality of programming.

How do I get the work-piece in the drawing faster?

CAD-Reader can do it; CAD-Reader is a tool for turning drawings directly into processing programs. NC production mainly adopts complex and abstract code for NC programming. only professionals can operate it. In the fact, Every skilled worker is professional, he can also take advantage of the rich experience accumulated in traditional machining to deal with difficult tasks, Although the approach is usually uneconomical. Therefore, it is necessary to find a way to enable these technical experts to effectively apply their experience to the production of NC machine tools.

HMI CAD-Reader software can implement the following functions:

(1) Converting the specified outline in a DXF drawing to an outline program in a specified programming format. For example, the drawings drawn by the commonly used drawing engineering software (Auto, CAD, CAXA, electronic chart, etc.) are saved in DXF format, and the program can be programmed directly by using CAD-Reader software. And you don't have to draw a graphical outline for the CAM program.

(2) Arrange the holes that need to be processed in the DXF drawings into the specified programming format, position definition, and outline procedures. Similarly, the files that are known to be holes are saved into DXF format, and the program can be programmed directly by using CAD-Reader software. You don't have to draw the hole again to make the CAM program. 


\section{PROCESS ANALYSIS}

CAD-Reader software generates processing programs in two cases:

A. generates contour programs: DXF outline drawings, G code outline procedures.

B. generates hole program: DXF hole drawing, G code, hole definition program.

\section{CAD-READER PROGRAMMING EXAMPLE ANALYSIS}

\subsection{DXF contour drawings $\longrightarrow$ G code profile}

(1) Open the L2-2.DXF parts drawing under the target folder

(2) Select "Setting" to enter setup menu. select "contour" profile editing program, click the options set: program definition into the profile type, contour plane, diameter / axis radius of information programming, the output of the number of decimal places, such as: G17, standard G code, programming radius. Confirm settings and return to main screen.

(3) click on the outline window where you want to zoom in. The cursor presses the left mouse button at the appropriate position and keeps it moving until the cursor is in place and the picture is adjusted to the right size. Before you click Cancel, select the enlarged view to restore the full display.

(4) Click to define the zero of the work-piece. Select the center of the part is at the center of the coordinate system, as shown in figure 1-1. Select the starting point for the elements in the outline of the cursor to the work-piece zero, the corresponding contour elements near the system automatically capture the contour elements displayed in green, click the left mouse button, the system there is zero symbols in the element at the starting point, and update the current coordinate numerical display.

(5) Defines an outline label enters an outline label and confirms it

Milling plane: AA

Milling 48×48×20 convex contour and smoothing: $\mathrm{BB}$

Milling hexagon convex contour and smoothing: CC

(6) Defines the origin and destination of an outline. For example, select the hexagon bump outline, move the cursor near the element, and display the element as yellow when the system captures it. Click the left mouse button to confirm the element. Move the cursor to the beginning or end of the element, and appear the red arrow, click the left mouse button, select the start or end of the element as the starting point of the outline, that is, the direction of the contour is clockwise or counterclockwise. When the outline element appears, you need to move the cursor to the corresponding element and click the left mouse button to confirm the selection. Select the outline clockwise until the contour is complete and closed to define the end of the contour. The method of contour cutting is progressive milling or reverse milling, which depends on the clockwise and counterclockwise selection of the contour. The selected outline is shown as green.

Boss contour:

Climb Milling $\longrightarrow$ Select the outline clockwise

Conventional Milling $\longrightarrow$ Select the outline counterclockwise

Groove contour:

Climb Milling $\longrightarrow$ Select the outline clockwise

Conventional Milling $\longrightarrow$ Select the outline counterclockwise

(7) Save the outline program, select program type and save, convert contour generated by the program through U disk or CF card or network transmission to the CNC system in use. Three formats are available (mpf, .spf, .arc). 
Table1 Step content

\begin{tabular}{|c|c|c|}
\hline Step & Step content, Cutting tools, cutting parameters & Process diagram \\
\hline 1 & $\begin{array}{l}\text { Milling plane: end milling cutter of } ø 12 \mathrm{~mm}, \mathrm{n}=2100 \mathrm{r} / \mathrm{min} \text {, } \\
\qquad \mathrm{F}=350 \mathrm{~mm} / \mathrm{min} \text {. }\end{array}$ & \\
\hline 2 & $\begin{array}{l}\text { Drilling } 4 \text { central holes: Centering drill of } \emptyset 10 \mathrm{~mm} \text {, } \\
n=3200 \mathrm{r} / \mathrm{min}, \mathrm{F}=200 \mathrm{~mm} / \mathrm{min} .\end{array}$ & \\
\hline 3 & $\begin{array}{l}\text { Drilling } 4 ø 7.8 \text { holes: Twist drill of } ø 7.8, \mathrm{n}=1400 \mathrm{r} / \mathrm{min} \text {, } \\
\qquad \mathrm{F}=140 \mathrm{~mm} / \mathrm{min} .\end{array}$ & \\
\hline 4 & $\begin{array}{l}\text { Milling } 48 \times 48 \times 20 \text { convex contour and smoothing: } \\
\text { end milling cutter of } ø 12 \mathrm{~mm}, \mathrm{n}=1300 \mathrm{r} / \mathrm{min}, \mathrm{F}=260 \mathrm{~mm} / \\
\mathrm{min} .\end{array}$ & \\
\hline 5 & $\begin{array}{l}\text { Milling hexagon convex contour and smoothing: } \\
\text { end milling cutter of } ø 12 \mathrm{~mm}, \mathrm{n}=1300 \mathrm{r} / \mathrm{min}, \mathrm{F}=260 \mathrm{~mm} / \\
\min .\end{array}$ & \\
\hline
\end{tabular}

\subsection{DXF hole drawing $\longrightarrow$ G code hole definition program}

The program of hole location is approximately the same as that of outline program. There are two differences:

(1) Select Setting to enter the settings menu, select "Drill holes", and edit the status of the hole processing program.

(2) Select the machining hole: at any position, move the cursor one by one processing hole, click the end position to select. Single row distribution holes, moving the cursor near the starting hole, which is shown as purple red. Click the left mouse button to confirm the selection, which is displayed as green. The moving cursor defines the direction of the column hole. The selected hole in the direction is shown as red. Click the left mouse button to confirm the selection. Move the cursor, select the hole you need to process, and click the left mouse button to confirm the selection. The hole is shown green, and the selected hole is yellow in that direction. Verify that the selected hole has been shown as a dashed line. Select $4 \varnothing 7.8 \mathrm{~mm}$ holes in turn.

\subsection{Custom parameterized programming}

In the preparation of numerical control programs, the usual practice is to use the operating system has already developed a good command, and then we will through the calculation and Simulation of the program to be used in advance prepared. However, in the process some castings we found some casting size cannot be determined accurately, therefore cannot give a specific numerical procedure, so we can only in the processing of each part to let the workers play parts of the actual value, and then change the program, because the program segment length, content and complex, the scene change prone to error. If the process room is re programmed, the program is complex, and seriously affect the processing cycle of parts. The larger the number of parts, the more serious the problem will be. How can we quickly and accurately transfer the required program into our processing equipment? This is the development of CNC functions to solve the main problem.

\section{CONCLUSIONS}

How do I move from drawings to work-pieces faster? SIEMENS profile editing software CAD-Reader is undoubtedly a tool for turning drawings directly into machining programs. With the help of CAD-Reader, the NC programming can be easily realized by NC programming personnel. It also allows experienced NC programmers to finish programming faster and better and improve work efficiency. 


\section{REFERENCES}

[1] Berenbach, Brian, Rayment, Taryn, 2010. The evaluation of a requirements engineering training program at siemens. Proceedings of the 16th IEEE International Requirements Engineering Conference, No.8, 21-27.

[2] H. Jiu, W.H. Chen, X.H. Li, 2014. SINUMERIK829D milling operations and programming. Mach Industry Press, Beijing.

[3] J.F. Shao, J. Li, N.Y. Shen, Y.Y. He, 2014. Development of the software for ball nut grinding process based on Siemens 840D System. Advanced Materials Research, Vol.945-949, 1645-1650.

[4] J.P. Dou, 2012. High processing quality control measures. Machine tool and hydraulic, Vol .40, No.4, 212-124.

[5] Q. Wang, 2015. Automatic programming and application of complex parts. Shandong Indu Tech, No.17, 207-208.

[6] Y.H. Liu, Y.C. Tang, Y.G. Zhang, 2012. Application of automatic programming in NC machining. Jiangsu Textile, No.4, 42-43.

[7] Y.Q. Liang, 2015. Design and implementation of NC automatic programming system based on DXF file. University of Chinese Academy of Sciences.

[8] Y.X. Xu, G.Q. Wang, 2015. Application analysis of AutoCAD technology in NC automatic programming. Eworld, No.9, 114.

[9] Z.P. Wang, Y. Feng, 2011. Applications of R Parameter and programming, Jump instructions in SIEMENSsystem. Advanced Materials Research, Vol.211-212,886-890.

[10] Z.T. Ni, L.H. Liao, 2013. Comprehensive application of CAD/CAM software in CNC milling machine / machining center operation. Occupation, No. 12, 82-83. 